\title{
Adjustable Contrast Enhancement Using Fast Piecewise Linear Histogram Equalization
}

\author{
Saleh Javadi \\ Department of Mathematics and \\ Natural Sciences, Blekinge Institute \\ of Technology (BTH), \\ Karlshamn 37435, Sweden \\ saleh.javadi@bth.se
}

\author{
Mattias Dahl \\ Department of Mathematics and \\ Natural Sciences, Blekinge Institute \\ of Technology (BTH), \\ Karlskrona 37179, Sweden \\ mattias.dahl@bth.se
}

\author{
Mats I. Pettersson \\ Department of Mathematics and \\ Natural Sciences, Blekinge Institute \\ of Technology (BTH), \\ Karlskrona 37179, Sweden \\ mats.pettersson@bth.se
}

\begin{abstract}
Histogram equalization is a technique to enhance the contrast of the image by redistributing the histogram. In this paper, a fast piecewise linear histogram equalization method is introduced based on an adjustable degree of enhancement and piecewise continuous transformation functions using frequencies of different grey-levels. This method aims to address and maximize the contrast enhancement of the image by stretching the entire spectrum. For this purpose, particular nodes (bins) on the histogram are simultaneously detected that in comparison with recursive methods, it requires less computational time. Then, the particular nodes are stretched using transformation functions to align with the reference nodes. The experimental results indicate that the performance of the proposed method is promising in terms of contrast enhancement. Moreover, this method preserves the texture of various regions in the image very well through the equalization process by using the degree of enhancement.
\end{abstract}

\section{CCS Concepts}

- Computing methodologies $\rightarrow$ Computer graphics $\rightarrow$ Image manipulation $\rightarrow$ Image processing.

\section{Keywords}

Histogram equalization; Contrast enhancement; Histogram modification; Image/video enhancement.

\section{INTRODUCTION}

Histogram is an important feature that describes the frequencies of different intensity levels of a digital image. Histogram equalization (HE) is an image enhancement technique based on the redistribution of the intensity levels to increase the contrast [1] It can be categorized into two classes of global and local histogram equalization. Global $\mathrm{HE}$ applies the transformation function once on the entire image, while local HE transforms

Permission to make digital or hard copies of part or all of this work for personal or classroom use is granted without fee provided that copies are not made or distributed for profit or commercial advantage and that copies bear this notice and the full citation on the first page. Copyrights for third-party components of this work must be honored. For all other uses, contact the Owner/Author.

ICIGP 2020, February 8-10, 2020, Singapore, Singapore.

(C) 2020 Copyright is held by the owner/author(s).

ACM ISBN 978-1-4503-7720-1/20/02.

https://doi.org/10.1145/3383812.3383830 intensity level of each pixel based on its neighborhood [2]. The traditional histogram equalization method has some drawbacks such as over-saturation, significantly changing the average luminance of the image, and lack of an adjustable degree of enhancement [3].

In recent years, several methods have been suggested to enhance the image contrast with minimizing the traditional HE shortcomings [2-8]. In order to preserve the brightness of the input image, some algorithms (e.g. multi-HE, brightness bi-HE, dualistic sub-image HE) have been presented that decompose the image into several sub-images and then apply traditional HE on each one [2-4]. Nilsson et al. [6] introduced a new global HE based on the Successive Mean Quantization Transform (SMQT) that hierarchically finds the mean value of the pixels to stretch the dynamic range of the histogram. Weighted Thresholded HE (WTHE) was proposed that modifies the histogram before equalization to protect the less-probable levels of histogram [7]. Further, a dynamic histogram specification was suggested that stretches the histogram within a specified range based on the histogram critical points to achieve contrast enhancement [8]. Hongbo and Xia [9] introduced Co-Occurrence matrix-based HE (COHE) that makes a trade-off output grey-level by using the cooccurred local information and global grey-level distribution. Histogram-Based Locality-Preserving Contrast Enhancement (HBLPCE) was proposed to preserve the locality of the histogram by solving an optimization problem [10].

In this paper, we proposed a contrast enhancement algorithm based on the specification of fast piecewise linear histogram equalization (FPLHE). This method contains computation of preprocessing parameters, node (bin) specification, and finally transformation function calculation. The proposed method successfully enhances the image contrast while the undesirable effects of the traditional $\mathrm{HE}$ are significantly minimized. In addition, due to the simplicity of the implementation of this method, the processing time is suitable for real-time applications. The rest of this paper is organized as follows. Section 2 describes the methodology of the proposed system in a progressive manner. The experimental results are presented and discussed in section 3 . Finally, the conclusion is provided in section 4 .

\section{METHODOLOGY}

Let's assume $\mathbf{X}=\{x(i, j)\}$ represents an input digital image where $x(i, j)$ denotes the intensity level of the pixel at $(i, j)$. The total number of pixels in the image is $T$ and the grey scale has $L$ digitized levels that are $\left\{X_{0}, X_{1}, \ldots, X_{L-1}\right\}$ and therefore, $\forall x(i, j) \in$ $\left\{X_{0}, X_{1}, \ldots, X_{L-1}\right\}$. The frequency of $X_{k}$ is achieved by 


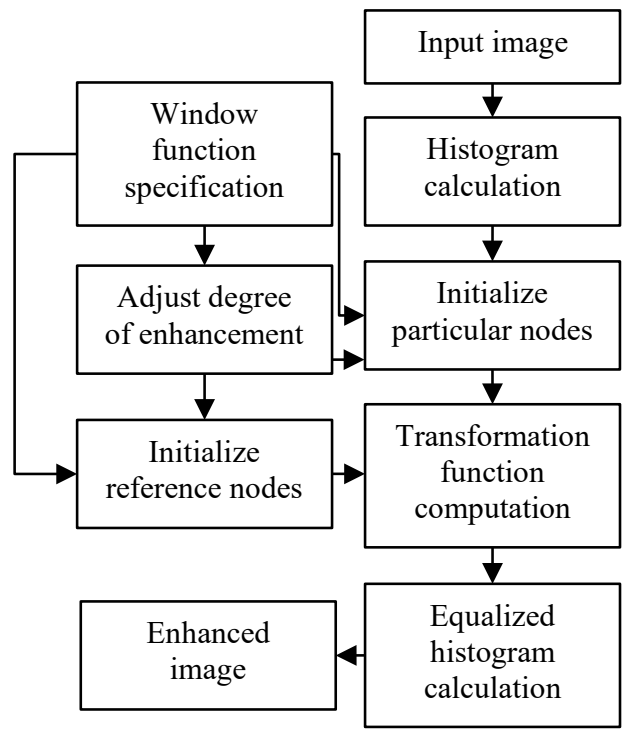

Figure 1. The block diagram of the proposed system.

$$
f\left(X_{k}\right)=\frac{t_{k}}{T}, \quad k=0,1, \ldots,(L-1)
$$

where $t_{k}$ is the number of pixels with the intensity level of $X_{k}$ in the image. Based on the frequencies, the relative cumulative frequency of the image is described as

$$
F\left(X_{k}\right)=\sum_{u=0}^{k} f\left(X_{u}\right), k=0,1, \ldots,(L-1)
$$

Let us assume that we want to enhance the image $\mathbf{X}=\{x(i, j)\}$ and $\mathbf{Y}=\{y(i, j)\}$ to be the enhanced image. The transformation from input image $\mathbf{X}$ to enhanced image $\mathbf{Y}$ can be shown as

$$
\mathbf{Y}=h(\mathbf{X})=\{h(x(i, j)) \mid \forall x(i, j) \in \mathbf{X}\}
$$

The main idea is to transform $x(i, j)$ in the input image $\mathbf{X}$ to the new value $y(i, j)$ in the output image $\mathbf{Y}$ where the contrast is enhanced. First, the degree of enhancement is selected that indicates how uniform the enhancement on the histogram will be applied. The higher the enhancement degree leads to the higher contrast enhancement of the final image. Based on the degree of enhancement, number of intensity levels on the histogram of the final image as the reference nodes are initialized. These reference nodes assure that the histogram of the enhanced image meets the uniform distribution to the extent of the degree of enhancement. In addition, particular nodes on the input image histogram are detected using relative cumulative frequencies of each intensity level and the degree of enhancement. Finally, multiple piecewise continuous transformation functions are computed and employed, in order to map the particular nodes to the reference nodes.

The block diagram of the proposed histogram equalization system is illustrated in Figure 1. We introduce $d \in\{1, \ldots, D\}$ as the degree of enhancement for a digital image with $2^{D-1}$ grey scale levels. For a standard 8-bit intensity level representation, the enhancement degree corresponds to $d \in\{1, \ldots, 9\}$. Consequently, the proposed method employs $\left(2^{d-1}+1\right)$ nodes or $2^{d-1}$ piecewise linear functions to perform histogram equalization on

\begin{tabular}{|c|c|c|c|c|c|c|}
\hline$d=1$ & $\left(2^{0}+1\right)$ nodes & $\mathrm{N}_{0}$ & & & & $\mathrm{~N}_{1}$ \\
\hline$d=2$ & $\left(2^{1}+1\right)$ nodes & $\mathrm{N}_{0}$ & & $\mathrm{~N}_{1}$ & & $\mathrm{~N}_{2}$ \\
\hline$d=3$ & $\left(2^{2}+1\right)$ nodes & $\mathrm{N}_{0}$ & $\mathrm{~N}_{1}$ & $\mathrm{~N}_{2}$ & $\mathrm{~N}_{3}$ & $\mathrm{~N}_{4}$ \\
\hline & $\vdots$ & & & & & \\
\hline$d=D$ & $\left(2^{D-1}+1\right)$ nodes & $\mathrm{N}_{0}$ & $\mathrm{~N}_{1}$ & & & $\mathrm{~N}_{2^{D-1}}$ \\
\hline
\end{tabular}
the entire grey scale spectrum. The degree of enhancement $d$ controls the strength of the contrast enhancement.
Figure 2. The tree of nodes on the histogram at each degree.

Figure 2 illustrates the relation between the degree of enhancement and the number nodes at each degree. At this stage, $\left(2^{d-1}+1\right)$ nodes are initialized on the spectrum of the enhanced image. These reference nodes are the destinations of the particular nodes of the input image after transformation. These nodes initialize the vector of reference nodes $\mathbf{N}_{\mathbf{Y}}=\left[N_{0}^{Y}, N_{1}^{Y}, \ldots, N_{M}^{Y}\right]$ that is placed on the histogram spectrum of the enhanced image as below.

$$
N_{m}^{Y}=Y_{\min }+m \frac{Y_{\max }-Y_{\min }}{2^{d-1}}, m=0, \ldots, 2^{d-1}
$$

where $m$ is the reference node number and $d$ is the degree of enhancement, $Y_{\min }$ and $Y_{\max }$ are the maximum and minimum intensity levels of the desired enhanced image that $\forall y(i, j) \in$ $\left\{Y_{0}, Y_{1}, \ldots, Y_{L-1}\right\}$. The default parameters for the proposed method are $Y_{\min }=0, Y_{\max }=255$, and $d=9$.

The vector of particular nodes $\mathbf{N}_{\mathbf{X}}=\left[N_{0}^{X}, N_{1}^{X}, \ldots, N_{M}^{X}\right]$ are initialized on the input image histogram that correspond to reference nodes $\mathbf{N}_{\mathbf{Y}}$ on the enhanced image. $N_{m}^{X}$ is computed based on the relative cumulative frequency of the input image $F\left(X_{k}\right)$ and the enhancement degree.

$$
N_{m}^{X}=X_{k} \text { if } F\left(X_{k}\right) \geq \frac{m}{2^{d-1}}, \quad m=0, \ldots, 2^{d-1}
$$

The transformation functions are computed to map the particular nodes on the input image histogram to the reference nodes on the enhanced image histogram. This procedure allows the simultaneous detection of nodes on both histograms and consequently calculation of the enhanced frequency $f\left(Y_{k}\right)$. The transformation is achieved using $2^{D-1}$ linear equations as below

$$
\lambda_{m}=\frac{N_{m+1}^{Y}-N_{m}^{Y}}{N_{m+1}^{X}-N_{m}^{X}}, \quad m=0, \ldots, 2^{d-1} .
$$

Thus,

$$
h\left(X_{k}\right)=Y_{k}=\lambda_{m}\left(X_{k}-N_{m}^{X}\right)+N_{m}^{Y}, \quad \begin{gathered}
m=0, \ldots, 2^{d-1} \\
S_{m} \leq X_{k} \leq S_{m+1} \\
k=0,1, \ldots,(L-1)
\end{gathered}
$$

In this process all the nodes on the original histogram is mapped to the equalized histogram. The advantage of this method is the simultaneous detection of the particular nodes on the histogram of the input image and furthermore the contrast enhancement that is inversely proportional to the intensity level frequency of the node in the input image. The frequency of the intensity levels of the enhanced image is obtained by

$$
f\left(h\left(X_{k}\right)\right)=f\left(X_{k}\right)
$$

This signifies that the distribution is equalized using a suitable degree of enhancement in accordance to the application in order to preserve the original textures of various regions in the image (see Figure 3). 


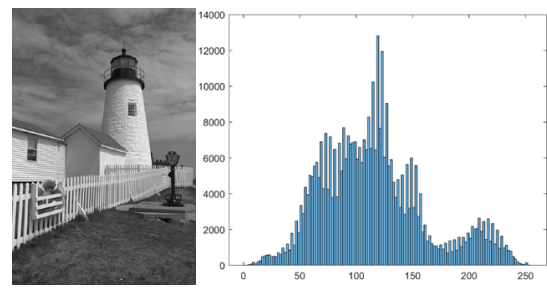

(a)

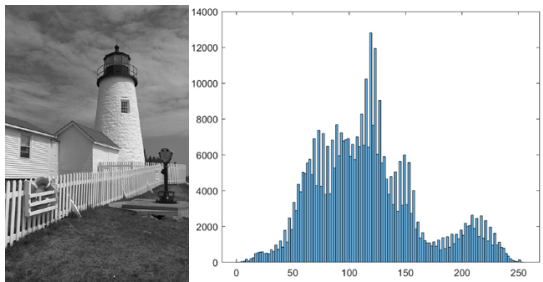

(b)

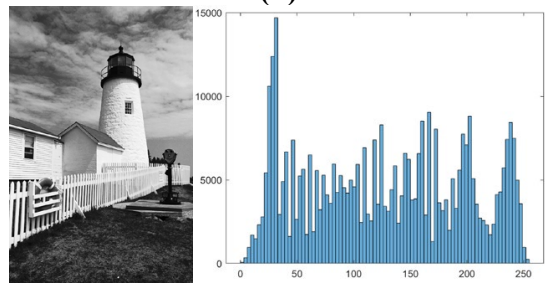

(e)

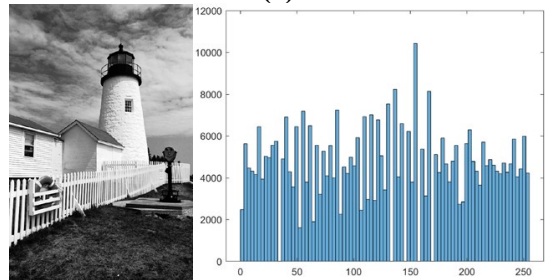

(h)

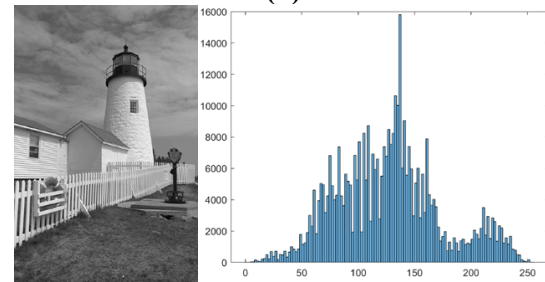

(c)
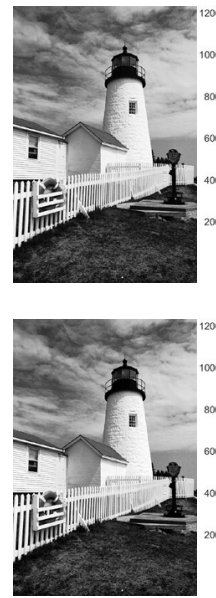

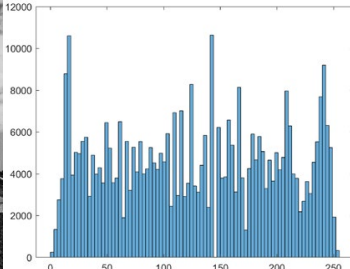

(f)

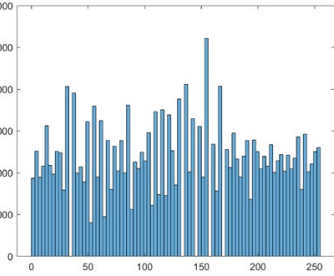

(i)

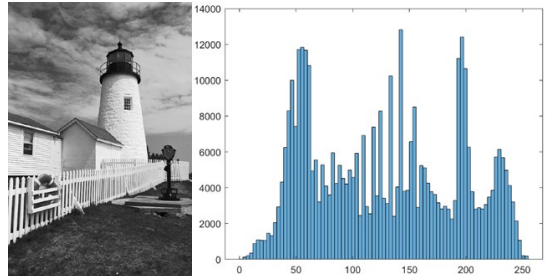

(d)

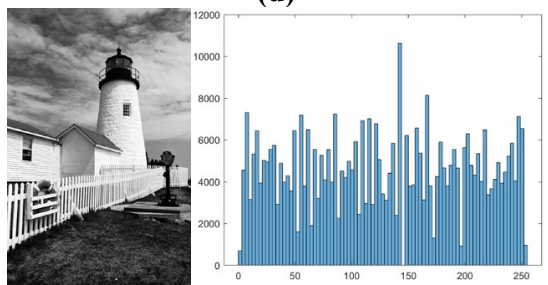

(g)
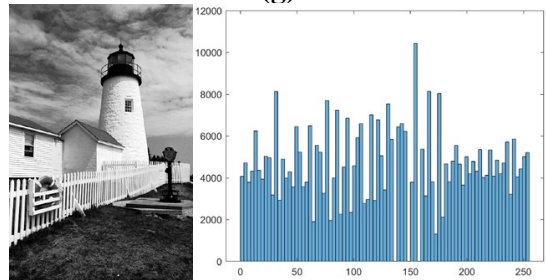

(j)

Figure 3. Histogram equalization (a) the original image histogram, (b) to (j) the enhanced image histogram with $d=1$ to $d=9$, respectively.

In practice, histogram equalization might cause some salt and pepper noise that is the result of over-enhancement in the less informative regions. Therefore, a window function can be applied on the original and the equalized histograms to achieve the enhanced histogram.

$$
Y_{k}=\left(1-w\left(X_{k}\right)\right) X_{k}+w\left(X_{k}\right) f\left(X_{k}\right)
$$

where $w\left(X_{k}\right)$ is the window function to control the level of enhancement based on the density of $X_{k}$.

\section{EXPERIMENTAL RESULTS AND DISCUSSION}

The proposed system has been realized on a standard computer platform in Microsoft Visual $\mathrm{C}++$ using OpenCV libraries. The objective is to enhance the contrast of the image while preserving the basic shape of the histogram and minimizing over-saturation. In this experiment, the proposed method is compared to the traditional $\mathrm{HE}$ and the SMQT using 24 images from Kodak database [11] and also 8 popular images including 'girl', 'jelly beans', 'Elaine', 'mandrill', 'Lenna', 'F-16', 'peppers', and 'Stream and bridge' from the USC-SIPI image database [12], as test images. The effectiveness of histogram equalization can be evaluated by measuring image brightness, image quality and image contrast.
The image brightness is evaluated using the absolute mean brightness error (AMBE) [13], which is the absolute difference of the brightness means of the original and enhanced image. The AMBE is calculated as,

$$
\mathrm{AMBE}=\left|\mu_{X}-\mu_{Y}\right|
$$

where $\mu_{X}$ is the brightness mean of the original image and $\mu_{Y}$ is the brightness mean of the enhanced image. The small AMBE suggests that the luminance level of the enhanced image is close to the original one.

The image quality in this paper is evaluated using the peak signalto-noise ratio (PSNR) which is an approximation to human perception of image quality [14]. The PSNR is computed as,

$$
\text { PSNR }=10 \log _{10} \frac{(L-1)^{2}}{\mathrm{MSE}}
$$

where $L$ is the intensity levels (e.g. 256) and MSE is mean square error that is defined as,

$$
\text { MSE }=\frac{1}{M N} \sum_{i=1}^{M} \sum_{j=1}^{N}|x(i, j)-y(i, j)|^{2}
$$

where $M$ and $N$ are the columns and rows of the image and $x(i, j)$ and $y(i, j)$ are the intensity levels at $(i, j)$ of input and enhanced 
Table 1. Performance evaluation of image brightness (AMBE) [13], image quality (PSNR) [14], and image contrast (EME) [15] using traditional HE [1], the SMQT [6] and the proposed method on the 24 images from Kodak database (G1) [11], and 8 popular images from USC-SIPI database (G2) [12].

\begin{tabular}{|c|c|c|c|c|c|}
\hline Metrics & Image & Original & HE & SMQT & FPLHE \\
\hline \multirow[t]{3}{*}{ AMBE (image brightness) } & G1 & - & 25.47 & 18.42 & 24.27 \\
\hline & G2 & - & 17.13 & 7.85 & 19.56 \\
\hline & Average & - & 23.38 & 15.78 & 23.09 \\
\hline \multirow[t]{3}{*}{ PSNR (image quality) } & G1 & - & 15.60 & 18.29 & 15.72 \\
\hline & G2 & - & 16.55 & 19.77 & 16.62 \\
\hline & Average & - & 15.84 & 18.66 & 15.94 \\
\hline \multirow[t]{3}{*}{ EME (image contrast) } & G1 & 8.23 & 16.09 & 14.12 & 16.26 \\
\hline & G2 & 6.39 & 15.06 & 12.76 & 15.26 \\
\hline & Average & 7.77 & 15.83 & 13.78 & 16.01 \\
\hline
\end{tabular}

images, respectively. The MSE parameter represents the average of the square differences between the corresponding pixels of the enhanced and the original images. The higher PSNR indicates that the enhanced image has been reconstructed better to match the original image.

However, the above parameters are not quite precise to evaluate the histogram equalization effectiveness on the enhanced image and they might be misleading. Since the output image without any enhancement can be interpreted as highly enhanced by using only AMBE and PSNR parameters that is obviously not correct. Therefore, another parameter measure of enhancement (EME) [15] is employed as follows,

$$
\mathrm{EME}=\frac{1}{D_{1} D_{2}} \sum_{c=1}^{D_{1}} \sum_{r=1}^{D_{2}} 20 \log \frac{I_{\text {max } r, c}^{w}}{I_{\text {min } ; r, c}^{w}}
$$

where the image is divided into $D_{1} \times D_{2}$ blocks and $I_{\text {max } ; r, c}^{w}$ and $I_{\text {min;r,c }}^{W}$ are the maximum and minimum grey-levels, in the block $w_{r, c}$, respectively. The high EME value indicates that the gap between the grey-levels is large and therefore the contrast in subimages and consequently in the image is high. If the essence of the histogram equalization is contrast enhancement, then the EME is the most suitable measure to evaluate this effect.

We applied histogram equalization using the traditional $\mathrm{HE}$, the SMQT and the proposed method (FPLHE) to 24 images from the Kodak database and 8 popular images from the USC-SIPI database. The performance of different methods then are measured by AMBE for image brightness, PSNR for image quality and finally EME for image contrast.

Table 1 summarizes the performance of each method using the values of AMBE, PSNR and EME. The images from Kodak database are shown as G1 and the images from the USC-SIPI database are shown as G2. As it is illustrated, the proposed method outperforms the traditional $\mathrm{HE}$ in terms of image brightness since it has a lower AMBE value. However, the SMQT achieves the minimum brightness error $($ AMBEsMQT $=15.78)$ and preserves the original luminance better than other methods. It is also observed that the image quality in comparison with the original image for human visual system is preserved higher by the SMQT $($ PSNRSMQT $=18.66$ ). The proposed system also performs better for image quality in comparison to the traditional HE. The
AMBE and PSNR parameters indicates that the processed image by the SMQT is the closest image to the original one in terms of brightness and pixel distance value. Although it does not necessary mean that enhancement is also superior to other methods. The EME parameter as described earlier, is a measure to evaluate the effectiveness of the histogram equalization regarding the contrast enhancement. The proposed method, FPLHE achieved the highest contrast enhancement value $($ EMEFPLE $=$ 16.01) that outperforms the SMQT and the traditional HE. The FPLHE shows also a significant contrast enhancement in comparison with the EME of the original image as well.

For visual quality assessment, number of pictures are presented and processed with different methods (see Figure 4). It is demonstrated that after contrast enhancement, the proposed method enhanced images with more details and comparatively preserved better the natural appearance of the original image. The darker regions of the image that the shadow casted strongly (e.g. top left and top right of the image), have been enhanced by our proposed method noticeably better than the traditional HE and SMQT. In addition, the lighter regions of the image (e.g. middle of the image) are also enhanced and preserved effectively after enhancement using the proposed method.

\section{CONCLUSION}

In this paper, a new histogram equalization method is introduced that aimed to enhance the contrast while preserving the basic shape of the histogram. The main idea is to implement piecewise continuous transformation functions using the frequencies of grey-levels and an adjustment degree. It is achieved by simultaneous detection and initialization of particular nodes (bins) on the input image's histogram and their corresponding reference nodes on the enhanced image's histogram based on the selected degree of enhancement. The proposed method successfully enhanced the contrast and preserved the natural appearance and the luminance of the original image. The experimental results demonstrate that the FPLHE outperforms the traditional HE and the SMQT methods in terms of contrast enhancement and achieves better visually images with less of typical shortcomings. Another advantage of the simultaneous node detection is the fast calculation of the transformation functions and consequently low processing time that makes this method suitable for real-time video enhancement. 


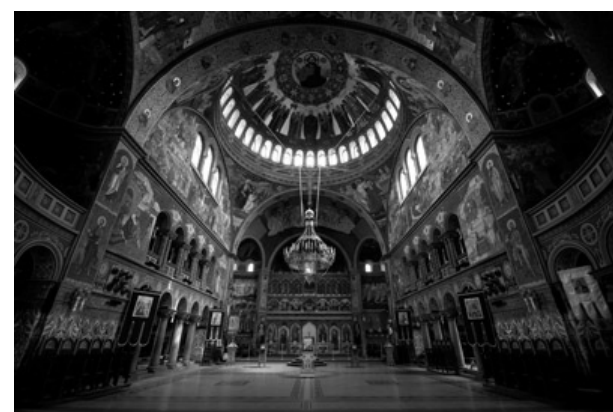

(a)

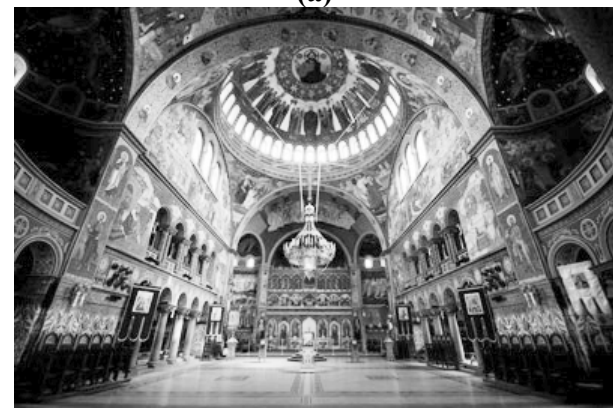

(c)

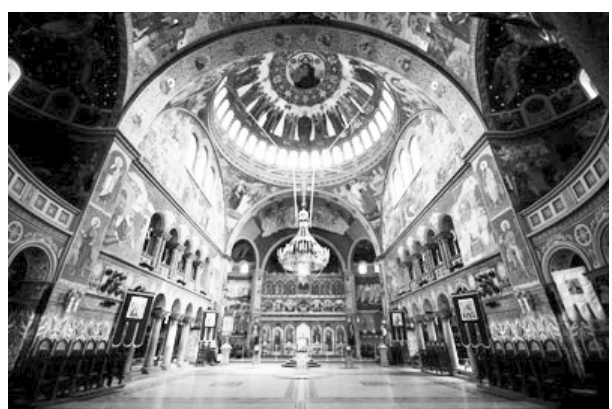

(b)

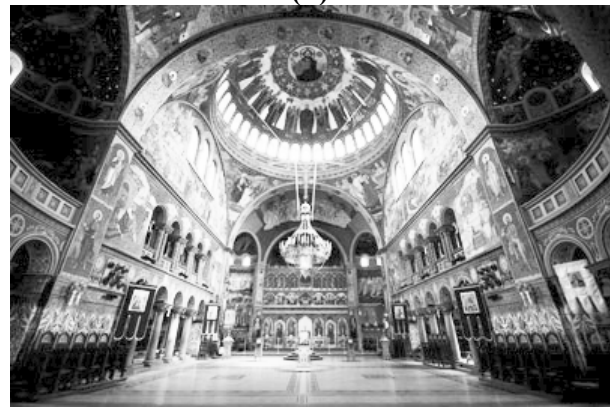

(d)

Figure 4. Enhancement contrast results (a) original image, (b) traditional HE, (c) SMQT, (d) the proposed method.

\section{REFERENCES}

[1] Gonzalez, R. C. and Woods, R. E. 2017. Digital Image Processing. $4^{\text {th }}$ edition, Pearson Education.

[2] Wang, Y., Chen, Q. and Zhang, B. 1999. Image enhancement based on equal area dualistic sub-image histogram equalization method. IEEE Trans. Consum. Electron. 45, 1 (Feb. 1999), 68-75. DOI= https://doi.org/10.1109/30.754419.

[3] Kim Y. T. 1997. Contrast enhancement using brightness preserving bi-histogram equalization. IEEE Trans. Consum. Electron. 43, 1 (Feb. 1997), 1-8. DOI= https://doi.org/10.1109/30.580378.

[4] Menotti, D., Najman, L., Facon, J. and Araujo A. D. A. 2007. Multi-histogram equalization methods for contrast enhancement and brightness preserving. IEEE Trans. Consum. Electron. 53, 3 (Aug. 2007), 1186-1194. DOI= https://doi.org/10.1109/TCE.2007.4341603.

[5] Kim, J. Y., Kim L. S. and Hwang, S. H. 2001. An advanced contrast enhancement using partially overlapped sub-block histogram equalization. IEEE Trans. Circuits Syst. Video Technol. 11, 4 (Apr. 2001), 475-484. DOI= https://doi.org/10.1109/76.915354.

[6] Nilsson, M., Dahl M. and Claesson I. 2005. Gray-scale image enhancement using the SMQT. IEEE International Conference on Image Processing 2005 (Genova, 2005). I933-6. DOI= https://doi.org/10.1109/ICIP.2005.1529905.

[7] Wang, Q. and Ward, R. K. 2007. Fast image/video contrast enhancement based on weighted thresholded histogram equalization. IEEE Trans. Consum. Electron. 53, 2 (May 2007), 757-764. DOI= https://doi.org/10.1109/TCE.2007.381756.

[8] Sun, C. C., Ruan, S. J., Shie M. C. and Pai, T. W. 2005. Dynamic contrast enhancement based on histogram specification. IEEE Trans. Consum. Electron. 51, 4 (Nov.
2005.), 1300-1305. DOI=

https://doi.org/10.1109/TCE.2005.1561859.

[9] Hongbo Y. and Xia, H. 2014. Histogram modification using grey-level co-occurrence matrix for image contrast enhancement. IET Image Process. 8, 12 (Dec. 2014), 782793. DOI= https://doi.org/10.1049/iet-ipr.2013.0657.

[10] Shin, J. and Park, R. H. 2015. Histogram-based localitypreserving contrast enhancement. IEEE Signal Process. Lett. 22, 9 (Sep. 2015), 1293-1296. DOI= https://doi.org/10.1109/LSP.2015.2399612.

[11] Kodak Lossless True Color Image Suite. Available at http://www.r0k.us/graphics/kodak/, accessed Nov. 2019.

[12] The USC-SIPI Image Database. Available at http://sipi.usc.edu/database/, accessed Nov. 2019.

[13] Chen, S. D. and Ramli, A. R. 2003. Minimum mean brightness error bi-histogram equalization in contrast enhancement. IEEE Trans. Consum. Electron. 49, 4 (Nov. 2003), 1310-1319. DOI= https://doi.org/10.1109/TCE.2003.1261234.

[14] Kim, M. and Chung, M. G. 2008. Recursively separated and weighted histogram equalization for brightness preservation and contrast enhancement. IEEE Trans. Consum. Electron. 54, 3 (Aug. 2008), 1389-1397. DOI= https://doi.org/10.1109/TCE.2008.4637632.

[15] Agaian, S. S., Panetta, K. and Grigoryan, A. M. 2001. Transform-based image enhancement algorithms with performance measure. IEEE Trans. Image Process. 10, 3 (Mar. 2001), 367-382. DOI= https://doi.org/10.1109/83.908502.

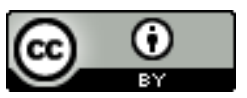

This work is licensed under a Creative Commons Attribution International 4.0 License. 